\title{
Palliative home care patients’ emergency department visits near end of life: decision making and outcomes
}

\author{
Charlotte Pooler, Joy Mekechuk, Domhnall O’Dochartaigh, Christa Desrochers, Jude \\ Spiers.
}

Background: Despite support and interventions in the community, some palliative patients have one or multiple Emergency (ED) visits near their end of life. People who are able to stay home are more likely to die at home or in hospice compared to those who are admitted to acute care. This study was to describe decisions and outcomes of palliative home care patients who go to emergency within the last 6 weeks of life in one metropolitan zone of Alberta Health Services.

Implementation: In the 2017-2018 fiscal year, there were 1874 palliative home care patients in the Edmonton Zone in urban (377) and rural/suburban (269) areas. Of these, 646 (34.4 \%) patients made 1033 known visits to the ED in their last 6 weeks of life. Charts of decreased patients were proportionally and randomly selected by area and CTAS score (CTAS1/2: 40; CTAS 3: 100: CTAS 4/5: 78), and purposefully selected for unusual events: died in the ED (21); left the ED (15); and admitted to critical care (6).

Results: Patients who went to ED were more likely to be male (59\%) and older than 65 years (65 \%). Most had cancer as a primary diagnosis (82.6\%). More 50 \% went to ED more than once. For the majority (74.3\%), the final ED visit was within 2 weeks of death; almost half were within 7 days (49.2\%). Primary presenting concerns were pain (24.9\%) and dyspnea (21.5\%). There was no known goal of care reported or documented in either the home care or hospital chart for $28.2 \%$ of patients. Goals or wishes of care were documented for $85 \%$ as reviewed or discussed in the ED, of whom $9 \%$ had their first order written and $47.8 \%$ had their order changed to align care with their wishes and illness. $44.6 \%$ spent 8 hours or less in ED; 21 patients died in the ED. Most patients (73.8\%) identified a preference to die at home or hospice; some were unknown (7.2 \%), others had not been discussed (17\%); 77.5\% died in hospital.

\section{Advice and Lessons Learned:}

1) This study highlights the ongoing opportunity to meet palliative care needs, including communication and collaboration between ED and home care.

2) Some patients presented urgently to home care near end of life; others were diagnosed in ED and referred to home care. Emergency clinicians have an important role in the management of symptoms and advance care planning in this population.

3) Additional anticipatory guidance may benefit those who present to the ED near end of life and enable them to attain their preferences to die at home or in hospice. 